\title{
Quintessence, Neutrino Masses and Unification of the Dark Sector
}

\section{Gennady Y. Chitov*}

Department of Physics, Laurentian University, Sudbury, ON, P3E 2 C6 Canada

E-mail: gchitov@laurentian.ca

The origin of the neutrino mass, dark matter (DM), and dark energy (DE) are among the most challenging problems of fundamental physics. We address these questions from analyses of the models where the neutrino mass is generated via Yukawa coupling to the quintessence field which represents the DE. It has been shown in a recent work on the toy model with a single Dirac fermion coupled to the quintessence that by choosing parameters of the DE potential to match the present DE density, the model allows to lock the neutrino mass at $m \sim 10^{-2} \mathrm{eV}$ and yields consistent estimates for other parameters of the Universe. To include the DM component into this framework we propose here to add the right-handed Majorana term(s) into Lagrangian with the quintessence-generated mass. As a results, the DE field is responsible for generation of the masses of the light active Majorana neutrinos as well as of the heavy sterile neutrinos. The latter are natural DM candidates.

The XXth International Workshop High Energy Physics and Quantum Field Theory September 24 - October 1, 2011

Sochi, Russia

\footnotetext{
*Speaker.
} 


\section{Introduction}

It appears now that consensus has being reached on our understanding of the composition of the Universe and fundamental issues demanding extensions of the standard model of particle physics. Roughly $75 \%$ of the Universe is comprised of dark energy (DE), about $22 \%$ of it is comprised of dark matter (DM), and only approximately 3\% corresponds to the contribution of the conventional (baryonic) matter. The present stage evolution of the Universe is governed by the dominant DE contribution, and the Universe experiences an accelerating expansion. The nature of the both DE and DM is still unknown, and this is probably one of the greatest mysteries of modern physics [1]. The origin of neutrino mass is also a fundamental problem for particle physics as well as for cosmology [2, 3]. Current upper limits on the sum of neutrino masses from cosmological observations are of the order of $1 \mathrm{eV}$, while neutrino oscillations give a lower bound of roughly $0.01 \mathrm{eV}[3]$. Even if at least ad hoc, minimalistically and intellectually unsatisfactory, the DE can be accounted for by adding a cosmological term to fit current observations, the existence of neutrino masses and DM particles definitely requires revisions of the standard model. There is a vast literature and a great interest in these issues. Due to space constraints we address the reader to "a short list" [1-6] where more references, especially on earlier work could be found.

In this paper we summarize and advance further our recent work [7]. It stems from the earlier proposal by Fardon et al [8]. In the approach [7] the origin of the neutrino mass is due to the dark energy in the form of quintessence. Both the DE density and the neutrino mass are determined by a single mass scale of the DE potential, and consistent estimates for other parameters of the Universe are obtained.

Here we propose how to incorporate the "third element of the puzzle", i.e, the DM component of the Universe within a common framework. For this end we analyze a quite straightforward extension of the "toy-model" of a single Dirac fermion considered in [7] by adding a right-handed Majorana fermion whose mass is due to the Yukawa interaction with the quintessence field. The Dirac-Majorana action for a single generation of neutrinos is known to be equivalent to two massive Majorana fermions. One of these, the (light) neutrino with mass $m_{a}$ decreasing in time can be identified with the conventional (active) neutrino, while the (heavy) sterile neutrino with growing mass $m_{s}$ is a natural candidate for the DM particle. The results and estimates we report below demonstrate that the theory is viable and consistent in unifying neutrino masses and the dark sector of the Universe.

\section{Toy Model: Dirac Fermion Coupled to the Ratra-Peebles Quintessence [7]}

The standard methods of general relativity and finite-temperature quantum field theory [9] were applied for fields in the flat Friedmann-Lemaitre-Robertson-Walker (FLRW) Universe. The scale factor $a(t)$ is governed by the Friedmann equations. The DE is modeled by the bosonic scalar field (quintessence) in the FLRW metric with the Euclidian action:

$$
S_{B}=\int_{0}^{\beta} d \tau \int a(t)^{3} d^{3} x\left[\frac{1}{2}\left(\partial_{\tau} \varphi\right)^{2}+\frac{1}{2 a^{2}}(\nabla \varphi)^{2}+U(\varphi)\right]
$$


where $\varphi=\varphi(\mathbf{x}, \tau)$ and $\hbar=c=k_{B}=1$. The analysis is restricted to the Ratra-Peebles quintessence potential:

$$
U(\varphi)=\frac{M^{\alpha+4}}{\varphi^{\alpha}}
$$

with $\alpha>0$. In this toy model a single massless neutrino is mimicked by the the Dirac Euclidian action

$$
S_{F}=\int_{0}^{\beta} d \tau \int a(t)^{3} d^{3} x \bar{\psi}(\mathbf{x}, \tau)\left(\gamma^{o} \frac{\partial}{\partial \tau}-\frac{l}{a} \gamma \cdot \nabla\right) \psi(\mathbf{x}, \tau) .
$$

The fermion and boson sectors of the Euclidian Lagrangian are interacting via the Yukawa coupling term:

$$
\mathscr{L}_{Y D}=g \varphi \bar{\psi} \psi .
$$

Note that the Ratra-Peebles potential $U(\varphi)$ does not have a nontrivial minimum, so the generation of the fermion mass is due to the coupling between the quintessence and fermions resulting in a nonzero average of the bosonic field: $m=g\langle\varphi\rangle$. (In the following the dimensionless Yukawa coupling is set $g=1$.) Evaluation of the grand partition function in the saddle-point approximation [7] yields the density of the thermodynamic potential

$$
\Omega(\varphi)=U(\varphi)+\Omega_{F}(\varphi)
$$

calculated at $\varphi=\langle\varphi\rangle$, where $\Omega_{F}$ is the standard density of the thermodynamic potential of the free Dirac field [9]. The average of the scalar field is determined by minimization:

$$
\left.\frac{\partial \Omega(\varphi)}{\partial \varphi}\right|_{\varphi=\langle\varphi\rangle}=U^{\prime}(\varphi)+g \rho_{c h}=0
$$

where we introduce the chiral fermionic condensate density:

$$
\rho_{c h}=\frac{2 m}{\pi^{2} \beta^{2}} \int_{\beta m}^{\infty} \frac{\left(z^{2}-(\beta m)^{2}\right)^{\frac{1}{2}}}{e^{z}+1} d z .
$$

Analysis of the extrema of the thermodynamic potential (2.6) reveals three phases of the coupled model.

1). Stable phase, $T>T_{m}$ : At high temperatures the equation (2.6) has two nontrivial solutions. The root indicated with a large dot in Fig. 1 (case a) corresponds to a global minimum of the potential. It is a thermodynamically stable state. In this phase $\Omega<0$, so the pressure $P=-\Omega$ is positive. In the high-temperature phase the fermionic mass is small:

$$
m \approx M\left(\sqrt{6 \alpha} \frac{M}{T}\right)^{\frac{2}{\alpha+2}} \propto T^{-\frac{2}{\alpha+2}},
$$

The fermionic contribution is dominant and the thermodynamic potential behaves to leading order as that of the ultra-relativistic fermion gas. Two masses in this model demonstrate opposite temperature dependencies. The quintessence field with the conventionally defined mass

$$
m_{\phi}^{2}=\left.\frac{\partial^{2} U(\varphi)}{\partial \varphi^{2}}\right|_{\varphi=\langle\varphi\rangle}
$$




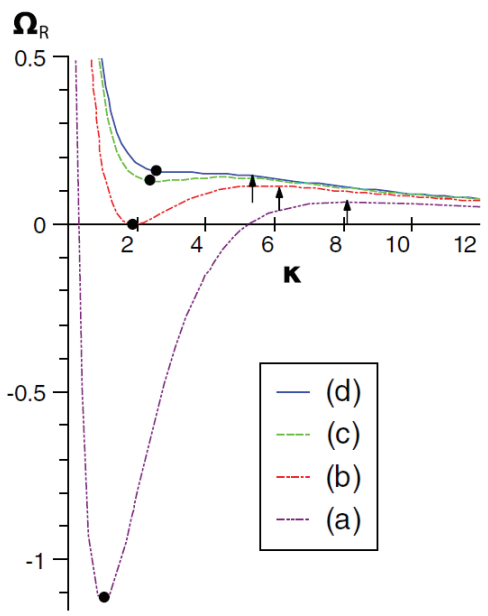

Figure 1: The dimensionless density of the thermodynamic potential $\Omega_{R} \equiv \Omega / M^{4}$ as a function of the dimensionless parameter $\kappa \equiv g \varphi / T$. The thermodynamically stable solutions of Eq. (2.6) indicated by the large dots correspond to the minima of the potential (curve a). The arrows indicate unstable solutions, corresponding to the maxima of the potential. The curve $\mathrm{b}$ is calculated at the temperature of metastability $T_{m}$. At lower temperature $T_{c}$ the local minimum becomes an inflection point of the potential (curve d), and the system gets unstable. This is a point of spinodal decomposition. Adapted from [7].

is "heavy" at high temperatures:

$$
m_{\phi} \approx \sqrt{\frac{\alpha+1}{6}} T, M / T \ll 1,
$$

however its mass decreases together with the temperature. In contrary, the fermionic mass $m$ monotonously increases with decreasing temperature.

Another non-trivial root of (2.6) corresponds to a thermodynamically unstable state (maximum of $\Omega$ indicated with an arrow in Fig. 1. There is a trivial third root $\varphi=\infty$ corresponding to the "doomsday" vacuum with a vanishing dark energy. In the high-temperature phase the doomsday vacuum $\Omega=0$ is a metastable state of the Universe.

2). Metastable phase, $T_{m}<T<T_{c}$ : At the temperature of metastability $T_{m}$ the thermodynamic potential has two degenerate minima $\Omega(\langle\varphi\rangle)=P(\langle\varphi\rangle)=\Omega(\infty)=0$. This is shown in Fig. 1 (case b). Below this temperature the two minima of the potential exchange their roles. The root indicated ny a large dot becomes a metastable state with $\Omega(\langle\varphi\rangle)>0$, i.e., with the negative pressure $P(\langle\varphi\rangle)<0$, while the stable state of the system corresponds to the true stable vacuum of the Universe $\Omega(\infty)=P(\infty)=0$. See Fig. 1 (case c). The state in the local minimum $\Omega(\langle\varphi\rangle)$ is analogous to a metastable supercooled liquid. We disregard here the exponentially small sphaleron contributions of the fermions hopping from the metastable state $\Omega(\langle\varphi\rangle)$ into the vacuum state $\Omega(\infty)=0$.

3). Phase transition at $T=T_{c}$ (spinodal decomposition): At the critical temperature

$$
T_{c} \approx \frac{M}{\Delta_{c}}
$$

two extrema of the potential merge into an inflection point. This is shown in Fig. 1 (case d). Here we define

$$
\Delta_{c}=\left(\frac{\sqrt{2}}{\alpha \pi^{3 / 2}} v^{v} e^{-v}\right)^{\frac{1}{\alpha+4}}, v=\alpha+\frac{5}{2} .
$$


From the viewpoint of equilibrium thermodynamics the model must undergo a first-order (discontinuous) phase transition and reach its thermodynamically stable (at $T<T_{c}$ ) phase corresponding to the doomsday vacuum $\Omega(\varphi=\infty)=P(\varphi=\infty)=0$. At the critical point the sound velocity and the compressibility vanish, thus the phase transition occurs through spinodal decomposition. During this transition the fermionic mass given at the critical point by

$$
m_{c} \approx \frac{v}{\Delta_{c}} M
$$

and the quintessence mass

$$
m_{\phi}^{c} \approx \sqrt{\alpha(\alpha+1)}\left(\frac{\Delta_{c}}{v}\right)^{\frac{\alpha+2}{2}} M
$$

both jump from the above values to their values in the vacuum state $m=\infty$ and $m_{\phi}=0$.

It is quite a nontrivial problem to analyze the time evolution of the Universe towards the new equilibrium vacuum state. In our work [7] we abandoned completely the thermodynamic or kinetic considerations below $T_{c}$ and resort to the solution of the equation of motion

$$
\ddot{\varphi}+3 H \dot{\varphi}+\frac{\partial \Omega}{\partial \varphi}=0 .
$$

together with the Friedmann equations. The parameters found at the critical point were used as the initial conditions for the dynamics at $T<T_{c}$. The numerical solution for the quintessence field $\varphi(t)$ from the critical point to the present time oscillates quickly (with the period $\tau \sim 10^{-27} \mathrm{Gyr}$ ) around the smooth ("mean value") solution $\bar{\varphi}(t)$. The latter is found analytically as

$$
\bar{\varphi}=\varphi_{c} \cdot\left(\frac{1+z_{c}}{1+z}\right)^{\frac{3}{\alpha+1}} .
$$

Relating the mean values with the physically relevant observable quantities, the key results are obtained analytically. To connect the only physical scale of the model $M$ with the observables, we set the current density of the quintessence field coupled to the fermions to be equal to the observable value of the dark energy, i.e., roughly $3 / 4$ of the critical density:

$$
\rho_{\varphi v, \text { now }}=\rho_{D E} \approx \frac{3}{4} \cdot \frac{3 H_{0}^{2}}{8 \pi G} \approx 31 \cdot 10^{-12} \mathrm{eV}^{4},
$$

and the combined baryonic and dark matter $\rho_{M \text {,now }} \approx \frac{1}{4} \cdot \frac{3 H_{0}^{2}}{8 \pi G}$. The exponent of the quintessence potential $\alpha$ is then the only parameter which can be varied. It is found that to get a realistic estimate for the present-time neutrino mass $m(t)=\bar{\varphi}(t)$, one needs a small $\alpha$. For instance, at $\alpha=0.01$ $m_{\text {now }} \approx 0.27 \mathrm{eV}$. The relative energy densities $\Omega_{\#} \equiv \rho_{\#} / \rho_{\text {tot }}$ as functions of redshift (or temperature) are plotted in Fig. 2. There we also indicate the critical point parameterized by the redshift $z_{\mathrm{c}}$ and the crossover point $z^{*}$. The latter is defined as the redshift at which the Universe starts its late-time acceleration, i.e., where $w_{\text {tot }} \equiv P_{\text {tot }} / \rho_{\text {tot }}=-\frac{1}{3}$. For the present time we find $w_{\text {tot }} \approx-\frac{3}{4}$.

Being successful in relating the neutrino mass to the DE, this toy model have two major flaws. First, it does not give a clue about (heavy) DM particles. Secondly, a more serious problem is that in order to get a small neutrino mass we need a small $\langle\varphi\rangle$. This makes the mass too sensitive to the local temperature and thus the existence of the fundamentally identical particles in the Universe very problematic. As we will see in the next section the seesaw mechanism in the extended model allows to resolve these two problems on the same footing. 


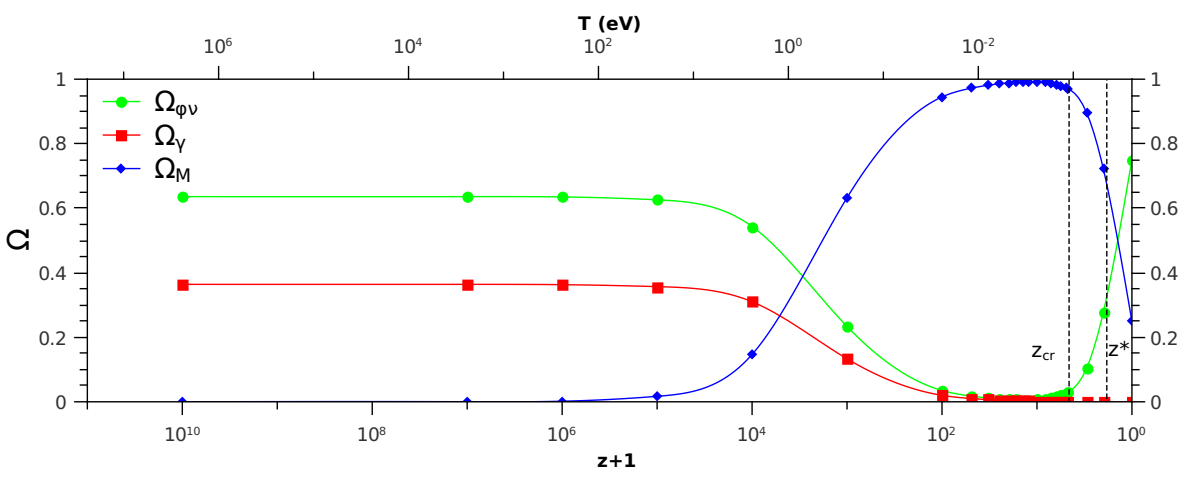

Figure 2: Relative energy densities plotted up to the current redshift (temperature, upper axis): $\Omega_{\varphi v}-$ coupled DE and neutrino contribution; $\Omega_{\gamma}$ - radiation; $\Omega_{M}$ - combined baryonic and dark matters. Parameter $M=2.39 \cdot 10^{-3} \mathrm{eV}(\alpha=0.01)$, chosen to fit the current densities, determines the critical point of the phase transition $z_{c} \approx 3.67$. The crossover redshift $z^{*} \approx 0.83$ corresponds to the point where the Universe starts its accelerating expansion. Adapted from [7].

\section{Extension of the toy model: Seesaw, Active and Sterile Majorana Neutrinos, Dark Energy}

To elucidate key points a theory needs to unify all three elements - neutrino, DM and DE within the same framework, we extend minimally the above toy model. For simplicity we take only one flavor of neutrinos. In addition to the Dirac mass term in the Lagrangian (2.4) written in terms of the left- and right-handed components of the Dirac field $\psi$ as $\mathscr{L}_{Y D}=m_{D} \bar{\psi}_{R} \psi_{L}+$ h.c., let us assume the existence of the right-handed Majorana neutrinos with the mass term $m_{R}$ :

$$
\mathscr{L}_{Y M}=\frac{1}{2} m_{R} \bar{\psi}_{R}^{c} \psi_{R}+\text { h.c. },
$$

where the charge conjugation is defined as $\psi_{R}^{c}=-l \gamma^{2} \psi_{R}^{*}$. The extensions of the standard model to include the Majorana mass term have been known for quite a while (for reviews and original references, see, e.g., [10, 11]), and more recent work pertinent to the present context in [4-6]). We take the standard assumption from the earlier work ([10, 11, 4-6] and more references there in), that the Dirac mass is due to the Yukawa coupling to the neutral Higgs scalar, i.e., $m_{D}=g_{D}\langle H\rangle$. We propose here that the origin of the Majorana neutrino mass is due to the coupling to the dark energy (quintessence), i.e., $m_{R}=g\langle\varphi\rangle$. To the best of our knowledge this was not studied before. The proposal closest to the present one is put forward by Shaposhnikov and co-workers [4, 5] in the framework of the so-called $v$ MSM extension of the standard model, where the right-handed neutrinos get their masses through the coupling to the inflaton field. ${ }^{1}$

Treatment of the fermionic action with the Dirac and Majorana terms is a well-known (seesaw) problem [10, 11]. The action can be brought to the diagonal form of the two Majorana fermions with positive masses:

$$
m_{s, a}=\frac{1}{2}\left(\sqrt{m_{R}^{2}+4 m_{D}^{2}} \pm m_{R}\right) .
$$

\footnotetext{
${ }^{1}$ It is quite plausible that the inflaton and quintessence represent the same physical field analyzed at the different regimes of the Universe evolution. In this sense the current proposal might turn out to be equivalent to the one of [5].
} 
Taking the Dirac mass as a fixed parameter, we treat the model of two Majorana fermions coupled to the quintessence along the lines of the problem with a single Dirac fermion [7]. In the saddlepoint approximation we get the thermodynamic potential (2.5) with the fermionic contribution from two Majorana fields and minimization equation

$$
U^{\prime}(\varphi)+\frac{1}{2} \frac{g}{\sqrt{m_{R}^{2}+4 m_{D}^{2}}}\left(m_{s} \rho_{c h}\left(m_{s}\right)-m_{a} \rho_{c h}\left(m_{a}\right)\right)=0,
$$

Let us now set the physically acceptable range of the model's parameters. We set the quintessence Yukawa coupling $g=1$ (this can always be done by rescaling of $M$ [7]) and take the mass of the active light Majorana neutrino at present time $m_{a}=10^{-2} \mathrm{eV}$. Since we don't know the Yukawa coupling $g_{D}$ between the Dirac and Higgs fields $(\langle H\rangle=174 \mathrm{GeV})$, we put $m_{D}$ in the range from the temperature of electroweak scale where the Higgs bosons acquire masses, i.e., $m_{D} \sim T_{E W} \sim 10^{2}$ $\mathrm{GeV}$ to the scale of light leptons $m_{D} \sim 1 \mathrm{MeV}$. Then the current mass of the heavy (sterile) Majorana neutrino is actually given by the quintessence $m_{s} \approx m_{R} \approx m_{D}^{2} / m_{a}$ and lies within $10^{15}-10^{5} \mathrm{GeV}$. Matching then the current Ratra-Peebles potential to the DE density (cf. Eq. (2.17)) we get the range $M \sim 5 \cdot 10^{2}-5 \mathrm{eV}$. For reader's convenience we put the model's parameters together with some other quantities in Table 1. Out of curiosity we also added there an entry with an ultralight Dirac mass $m_{D}=1 \mathrm{KeV}$.

At high temperatures $T>T_{E W}$ when $m_{D}=0$ the model is in a stable phase whose parameters (up to a factor 2) coincide with that of the above case of a single Dirac fermion [7]. The mass of active neutrino $m_{a}=0$, while the sterile neutrino is light and relativistic $m_{s}=m_{R} \approx \mathscr{O}(1)$. $M(M / T)^{2 /(\alpha+2)}$.

At $T<T_{E W}$ behavior of the model with Majorana neutrinos demonstrates some new qualitative features comparatively to the case [7] due to: (i) the new mass scale $m_{D}$ which sets in below $T_{E W}$; (ii) decreasing $m_{a}$ with growing quintessence. The Dirac mass brings in a new critical temperature

$$
T_{c D}=\frac{2}{5} m_{D}
$$

below which the sign of the second term in the minimum equation (3.3) is always negative and no nontrivial solution for decreasing quintessence potentials exists. ${ }^{2}$

The evolution of the model below $T_{E W}$ towards the critical point of the first order phase transition is qualitatively similar to that shown in Fig. 1. The critical temperature where the maximum and minimum of the thermodynamic potential merge into an inflection point is found along with the critical value of the mass $m_{R}$ :

$$
T_{c} \approx T_{c D}\left(1+\zeta_{\alpha}\left(\frac{M}{m_{D}}\right)^{2}\right), \quad m_{R}^{c} \approx \vartheta_{\alpha} M
$$

where the numbers $\zeta_{\alpha} \sim \vartheta_{\alpha} \sim \mathscr{O}(1)$. For $\alpha=1$ we find $\zeta_{1} \approx 2.86$ and $\vartheta_{1} \approx 6.42$. As one can see from Table 1 in the physically interesting range of parameters $T_{c} \approx T_{c D}$, so the critical temperature is determined in fact solely by the Dirac mass $m_{D}$ (compare to Eq. (2.11)), while the quintessence at $T_{c}$

\footnotetext{
${ }^{2}$ For large $m_{D} \sim T_{E W}$ this opens an interesting opportunity for the future work to explore trial DE potentials other than the Ratra-Peebles one.
} 


\begin{tabular}{|c|c|c|c|}
\hline $\begin{array}{l} \\
M \\
m_{D}\end{array}$ & $\begin{array}{l}T \geq T_{E W} \sim 10^{2} \mathrm{GeV} \\
m_{R}=m_{s} \sim M\left(\frac{M}{T}\right)^{2 /(\alpha+2)} \\
m_{a}=m_{D}=0\end{array}$ & $\begin{array}{l}T=T_{c} \approx 0.4 m_{D} \\
m_{R}^{c} \approx 6.42 M \\
m_{s, a}^{c} \approx m_{D} \pm \frac{1}{2} m_{R}^{c}\end{array}$ & $\begin{array}{l}T=T_{\text {now }} \\
m_{R} \approx m_{s} \approx m_{D}^{2} / m_{a} \\
m_{a}-\text { fixed }\end{array}$ \\
\hline $\begin{array}{l}5 \cdot 10^{2} \mathrm{eV} \\
10^{2} \mathrm{GeV}\end{array}$ & $\begin{array}{ll}m_{R} & \leq 10^{-3} \mathrm{eV} \\
m_{s} & \leq 10^{-3} \mathrm{eV} \\
m_{a} & 0\end{array}$ & $\begin{array}{l}3 \mathrm{KeV} \\
10^{2} \mathrm{GeV} \\
10^{2} \mathrm{GeV} \\
\end{array}$ & $\begin{array}{l}10^{15} \mathrm{GeV} \\
10^{15} \mathrm{GeV} \\
10^{-2} \mathrm{eV} \\
\end{array}$ \\
\hline $\begin{array}{l}5 \mathrm{eV} \\
1 \mathrm{MeV}\end{array}$ & $\begin{array}{l}m_{R} \leq 10^{-6} \mathrm{eV} \\
m_{s} \leq 10^{-6} \mathrm{eV} \\
m_{a} \quad 0\end{array}$ & $\begin{array}{l}30 \mathrm{eV} \\
1 \mathrm{MeV} \\
1 \mathrm{MeV}\end{array}$ & $\begin{array}{l}10^{5} \mathrm{GeV} \\
10^{5} \mathrm{GeV} \\
10^{-2} \mathrm{eV}\end{array}$ \\
\hline $\begin{array}{l}0.3 \mathrm{eV} \\
1 \mathrm{KeV}\end{array}$ & $\begin{array}{l}m_{R} \leq 10^{-9} \mathrm{eV} \\
m_{s} \leq 10^{-9} \mathrm{eV} \\
m_{a} \quad 0\end{array}$ & $\begin{array}{l}2 \mathrm{eV} \\
1 \mathrm{KeV} \\
1 \mathrm{KeV}\end{array}$ & $\begin{array}{l}10^{8} \mathrm{eV} \\
10^{8} \mathrm{eV} \\
10^{-2} \mathrm{eV}\end{array}$ \\
\hline
\end{tabular}

Table 1: Neutrino masses at different temperatures $(\alpha=1)$ for three choices of the Dirac mass $m_{D}$. The current active neutrino mass is set $m_{a}=10^{-2} \mathrm{eV}$ and the quintessence potential scale $M$ is chosen to match the current DE density. All the parameters in the table are defined in the text.

is controlled by the Ratra-Peebles scale $M$, similar to Eq. (2.13). The quintessence field being heavy at high temperatures $m_{\phi} \sim T$, becomes lighter with cooling of the Universe, reaching $m_{\phi}^{c} \sim M$ at $T_{c}$, and vanishes below $T_{c}$. We have checked that the sound velocity and the compressibility vanish at $T_{c}$, thus this the spinodal decomposition point and the transition occurrs via rapidly growing density fluctuations below $T_{c}$.

\section{Discussion: Realistic Extension of the Standard Model, Dark Matter and Dark Energy}

It appears that the above model of Majorana neutrinos interacting with the quintessence and getting their masses from DE is consistent, and it encourages for further work on it. But we have to stress that below the phase transition the model is probed so far only by consistency checks between various parameters $\left(\rho_{D E}, m_{a}, m_{s}\right)$ and observables. Analysis of the equation of motion (2.15) for the quintessence field and/or by using more general methods of kinetics of the first-order phase transition is warranted.

However, the model itself needs to be more realistic. It seems to us that the so-called $v$ MSM [4. 5] is the right model which has already properties needed. This is an extension of the standard model where three additional right-handed Majorana gauge singlets for each of the neutrino flavors are added. With the model's available fitting parameters it is possible [4] to get along with the three light active neutrinos also the three sterile neutrinos with consistent abundances, masses, and life times. ${ }^{3}$ One of the sterile neutrinos with the life time comparable to the age of the Universe has a mass in the $\mathrm{KeV}$ range and is an excellent "warm" DM candidate, while the other short-lived heavy neutrinos have their masses in the GeV range and are decaying DM particles. The results presented here allow us to anticipate that using the quintessence field for generating the righthanded Majorana massive terms (3.1) in this extended standard model would also allow to obtain

\footnotetext{
${ }^{3}$ In addition this model also provides a mechanism for baryon asymmetry of the Universe.
} 
the observable DE density and the slow-rolling quintessence dynamics with $w_{\varphi} \equiv P_{\varphi} / \rho_{\varphi} \approx-1$. This study is currently work in progress.

\section{Acknowledgments}

I am thankful to my co-authors T. August, A. Natarajan and especially to T. Kahniashvili for collaboration on our paper [7]. I also thank A. Dolgov and M. Shaposhnikov for correspondence. I am very grateful to $\mathrm{O}$. Chkvorets for many helpful discussions and bringing important references to my attention. The author acknowledges financial support from the Natural Science and Engineering Research Council of Canada (NSERC) and the Laurentian University Research Fund (LURF).

\section{References}

[1] For reviews and more references, see, e.g., E. J. Copeland, M. Sami and S. Tsujikawa, Dynamics of dark energy, Int. J. Mod. Phys. D 15 (2006) 1753 [hep-th/0603057]; L. Amendola and S. Tsujikawa, Dark Energy: Theory and Observations, Cambridge University Press, Cambridge 2010.

[2] A. D. Dolgov, Cosmology and Neutrino Properties, Phys. Atom. Nucl. 71 (2008) 2152 [arXiv:0803.3887 [hep-ph]].

[3] J. Lesgourgues and S. Pastor, Massive neutrinos and cosmology, Phys. Rept. 429 (2006) 307 [astro-ph/0603494].

[4] T. Asaka and M. Shaposhnikov, The VMSM, dark matter and baryon asymmetry of the universe, Phys. Lett. B 620 (2005) 17 [hep-ph/0505013]; T. Asaka, S. Blanchet and M. Shaposhnikov, The vMSM, dark matter and neutrino masses, Phys. Lett. B 631 (2005) 151 [hep-ph/0503065].

[5] F. L. Bezrukov and M. Shaposhnikov, The Standard Model Higgs boson as the inflaton, Phys. Lett. B 659 (2008) 703 [arXiv:0710.3755 [hep-th]]; M. Shaposhnikov and I. Tkachev, The vMSM, inflation, and dark matter, Phys. Lett. B 639 (2006) 414 [arXiv:hep-ph/0604236].

[6] B. Kayser, Neutrino Mass, Mixing, and Flavor Change, Springer Tracts in Modern Physics, 190 (2003) 1-24 [hep-ph/0211134].

[7] G.Y. Chitov, T. August, A. Natarajan, T. Kahniashvili, Mass Varying Neutrinos, Quintessence, and the Accelerating Expansion of the Universe, Phys. Rev. D 83 (2011) 045033 [arXiv:0911.1728 [astro-ph.CO]].

[8] R. Fardon, A. E. Nelson and N. Weiner, Dark energy from mass varying neutrinos, JCAP 0410 (2004) 005 [astro-ph/0309800].

[9] J.I. Kapusta and C. Gale, Finite-Temperature Field Theory: Principles and Applications, Second Edition, Cambridge University Press, Cambridge 2006.

[10] T.-P. Cheng and L.-F. Li, Gauge Theory of Elementary Particle Physics, Oxford University Press, New York 1984.

[11] S. M. Bilenky and S. T. Petcov, Massive neutrinos and neutrino oscillations, Rev. Mod. Phys. 59 (1987) 671. 